INVESTIGACIONES

\title{
Adaptación socioemocional en escolares: evaluación de un instrumento nuevo en la provincia de Concepción*
}

\author{
Students' socio-emotional adaptation: assessment of \\ a new instrument in Concepción city \\ Adaptação socioemocional em estudantes: avalição de um \\ novo instrumento na província de Concepción \\ M. Elena Mathiesen ${ }^{1}$, José Merino ${ }^{2}$, \\ Ginette Castro ${ }^{3}$, Olga Mora ${ }^{4}$, Gracia Navarro \\ ${ }^{1}$ Departamento de Sociología y Antropología, \\ Fac. de Ciencias Sociales, Universidad de Concepción. 041-2203105.mmathies@udec.cl \\ ${ }^{2}$ Departamento de Sociología y Antropología, Fac. de Ciencias Sociales, \\ Universidad de Concepción. 041-2203044. jmerino@udec.cl \\ ${ }^{3}$ Departamento de Idiomas Extranjeros, Fac. de Humanidades y Arte, \\ Universidad de Concepción. 041-2204540. gcastro@udec.cl \\ ${ }^{4}$ Departamento de Trabajo Social, Fac. de Ciencias Sociales, \\ Universidad de Concepción.041-2204403. omora@udec.cl \\ ${ }^{5}$ Departamento de Psicología, Fac. Ciencias Sociales, \\ Universidad de Concepción. 041-2203037. gnavarro@udec.cl
}

\begin{abstract}
RESUMEN
Se presentan los resultados psicométricos de un instrumento creado en 2007 para evaluar adaptación socioemocional en niños/as (mayores de 10 años) y jóvenes. Además se hace una evaluación, por ítem, de la adaptación socioemocional de estos estudiantes. Este instrumento se basó en una investigación cualitativa del equipo de sicólogos de Talentos UdeC. Se aplicó a una muestra de 1010 alumnos/as preseleccionados por sus profesores como con talento académico destacado, desde quinto básico a tercero medio, en cuatro periodos, desde 2007 a 2010 . El análisis factorial muestra tres factores, algo diferentes a las tres subescalas originales, cuatro de los 27 ítems deben ser eliminados, quedando el cuestionario de 23 ítems. La confiabilidad del instrumento es alta, los indicadores de validez son adecuados, por tanto puede ser usado, en nuestra realidad, para medir adaptación socioemocional en niños/as y jóvenes. La adaptación socioemocional observada es en general alta.
\end{abstract}

Palabras clave: adaptación socioemocional, alumnos con talento, psicometría.

\begin{abstract}
This study illustrates psychometrical findings from a 2007 instrument applied to measure socio-emotional adaptation in children over 10 years old. An item-evaluation of the socio-emotional adaptation of these students is also included. This scalar instrument was developed in prior qualitative research performed by the UdeC Talent Program team of psychologists. It was applied to a sample of 1010 students pre-selected by their teachers as gifted student, grades 5-11, in four cohorts from 2007 to 2010 . Factorial analysis indicated three factors slightly different from the original three subscales; four out of 27 items had to be eliminated, reducing to 23 the final number of items in the questionnaire. Since scale reliability was high and the validity indicators were adequate, the instrument has been shown to satisfactorily measure children's socio-emotional adaptation to the Chilean social reality. The socio-emotional adaptation observed was moderately high.
\end{abstract}

Key words: socio-emotional adaptation, students with talent, psychometrics.

* Este trabajo se inserta en el Proyecto Fondecyt No 1100260 (Mathiesen, Merino, Mora, Navarro y Castro, 2010-2011). 


\section{RESUMO}

Apresentam-se os resultados psicométricos de um instrumento criado em 2007 para avaliar a adaptação socioemocional de crianças (acima de 10 anos) e jovens. Além disso, faz-se uma avaliação, por item, da adaptação socioemocional desses estudantes. O instrumento baseia-se em uma pesquisa qualitativa da equipe de psicólogos do Programa de Talentos, UdeC, e foi respondido em quatro oportunidades, de 2007 até 2010, por 1010 estudantes do quinto ano do Ensino Fundamental ao terceiro ano do Ensino Médio, previamente classificados por seus professores como estudantes com desempenho acadêmico satisfatório. A análise fatorial revela três itens levemente diferentes das três subescalas originais. Quatro dos 27 itens foram eliminados, restando 23. A confiabilidade no instrumento é alta, os indicadores de legitimidade são apropriados e ele pode ser utilizado em nossa realidade para avaliar a adaptação socioemocional de crianças e jovens. A adaptação socioemocional observada neste estudo foi predominantemente alta.

Palavras chave: adaptação socioemocional, estudantes com desempenho acadêmico satisfatório, psicometria.

\section{INTRODUCCIÓN}

Existe evidencia acerca de que la adaptación socioemocional en escolares con alto talento académico podría verse afectada por el ambiente escolar que no valora sus capacidades especiales y los obliga a ir al ritmo promedio, lo que podría ser dañino no solo para el cultivo del talento mismo, sino también para el desarrollo integral de la personalidad (Bralic y Romagnoli 2000). El programa Talentos U de C decidió el año 2006 incorporar esta variable en test aplicados a los alumnos que postulan al Programa; para esto creó el instrumento cuyos resultados presentamos en este trabajo. Al referirnos a talento académico destacado lo hacemos siguiendo a Gagné (2000) quien sostiene que el talento alude al dominio destacado de habilidades, destrezas y conocimientos sistemáticamente desarrollados en al menos un campo de la actividad humana, en este caso el área académica, en grado que sitúa al individuo dentro del $10 \%$ superior del grupo de su misma edad que cultiva o ha cultivado ese campo.

Encontraremos por tanto en este artículo los resultados psicométricos de la aplicación de este instrumento en niños y jóvenes alumnos de establecimientos educacionales, preseleccionados por sus profesores como con talento académico destacado, que cursaban desde quinto año básico hasta tercero medio. Se presentan también los promedios de adaptación totales, por subescala y por ítems.

Se trata de una escala tipo Likert, construida a partir de los resultados de una investigación cualitativa realizada en una muestra de 10 psicólogos clínicos infanto-juveniles que se desempeñaban profesionalmente en la provincia de Concepción, a los cuales se les consultó por conductas posibles de ser autorreportadas, que reflejan adaptación socioemocional.

Siguiendo el modelo conceptual del Inventario de Adaptación de Conducta (IAC) de M.V. de la Cruz y A. Cordero (1988) se seleccionó comportamientos de las tres áreas a las que los escolares deben adaptarse: la escolar, la familiar y la personal; y que además reflejaran habilidades sociales, emocionales y cognitivas.

Con esta base se construyó la escala que quedó formada por 27 ítems, con 5 alternativas de respuesta (totalmente en desacuerdo, un punto; en desacuerdo, dos; ni de acuerdo ni en desacuerdo, tres; de acuerdo, cuatro y totalmente de acuerdo, cinco). Del total de ítems, trece tienen formato invertido. Los 27 ítems seleccionados se distribuyeron en tres subescalas: Habilidades sociales, con 11 ítems. Habilidades emocionales, también con 11 ítems, y Habilidades cognitivas, con cinco ítems. 
Las preguntas de investigación que espera responder este trabajo son: ¿Cuáles son las dimensiones que evalúa este cuestionario? ¿Este instrumento es confiable? ¿El cuestionario presenta criterios de validez? Y por último ¿Cuál es el nivel de adaptación de los niños, niñas y jóvenes de la muestra? Así, el propósito es demostrar que el cuestionario de adaptación socioemocional creado por Navarro et al. ${ }^{1}$ es confiable y válido para ser aplicado en niños/as y jóvenes chilenos.

Los objetivos específicos son: realizar un análisis factorial de las dimensiones de la escala, evaluar su confiabilidad, inspeccionar su validez y mostrar la adaptación socioemocional, global y pormenorizadamente, en los escolares de la muestra.

\section{METODOLOGÍA}

\subsection{UNIVERSO Y MUESTRA}

El universo de esta investigación estuvo compuesto por todos los alumnos/as, de la provincia de Concepción, preseleccionados por sus profesores como candidatos al Programa Talentos $\mathrm{U}$ de $\mathrm{C}$ por tener talento académico destacado. La muestra quedó conformada por 1010 de estos estudiantes, preseleccionados entre los años 2007 y 2010 y que rindieron pruebas de selección.

\subsection{CARACTERÍSTICAS DE LA MUESTRA}

El $77 \%$ de los integrantes de la muestra provenían de colegios municipales, el $8 \%$ de particulares subvencionados y el $15 \%$ de particulares pagados (ver Tabla 1).

\section{Tabla 1}

Frecuencias absolutas de nominados/as de la muestra según tipo de sostenedor y año de preselección

\begin{tabular}{|l|r|r|r|r|c|}
\hline Sostenedor & 2007 & 2008 & 2009 & 2010 & Totales \\
\hline Municipal & 110 & 466 & 28 & 173 & $777(77 \%)$ \\
\hline Subvencionado & 62 & 15 & 4 & 1 & $82(8 \%)$ \\
\hline Particular & 0 & 9 & 90 & 52 & $151(15 \%)$ \\
\hline Totales & 172 & 490 & 122 & 226 & $1010(100 \%)$ \\
\hline
\end{tabular}

En la Tabla 2 se presenta la distribución de la muestra por nivel educacional y por año. Se puede apreciar que la mayoría (52\%) de los nominados eran de sexto básico, seguido del $21 \%$ de séptimo. El rango etario se mueve entre 9 y 19 años con un promedio de 11.9.

$1 \quad$ No existen publicaciones 
Tabla 2

Frecuencias absolutas de nominados/as de la muestra según nivel educacional y año de preselección

\begin{tabular}{|l|r|r|r|r|c|}
\hline Nivel & 2007 & 2008 & 2009 & 2010 & \multicolumn{1}{c|}{ Totales } \\
\hline Quinto & 0 & 0 & 18 & 1 & $19(2 \%)$ \\
\hline Sexto & 120 & 254 & 18 & 129 & $521(52 \%)$ \\
\hline Séptimo & 8 & 164 & 14 & 31 & $217(21 \%)$ \\
\hline Octavo & 10 & 0 & 14 & 6 & $30(3 \%)$ \\
\hline $1^{\text {o Medio }}$ & 12 & 70 & 26 & 16 & $124(12 \%)$ \\
\hline $2^{\text {o Medio }}$ & 11 & 0 & 19 & 8 & $38(4 \%)$ \\
\hline $3^{\text {o Medio }}$ & 11 & 2 & 13 & 35 & $61(6 \%)$ \\
\hline Totales & 172 & 490 & 122 & 226 & $1010(100 \%)$ \\
\hline
\end{tabular}

Como se puede observar en la tabla 3 , los alumnos integrantes de la muestra proceden, en diversas proporciones, de todas las comunas de la provincia de Concepción; como es natural, están representadas en mayor proporción las comunas más grandes como Talcahuano y Concepción, seguidas de Chiguayante, Lota, Hualpén y San Pedro.

\section{Tabla 3}

Frecuencias absolutas de nominados/as de la muestra según comuna y año de preselección

\begin{tabular}{|l|r|r|r|r|c|}
\hline Comuna & 2007 & 2008 & 2009 & 2010 & Totales \\
\hline Concepción & 55 & 3 & 48 & 34 & $140(14 \%)$ \\
\hline Talcahuano & 1 & 173 & 14 & 85 & $273(27 \%)$ \\
\hline Chiguayante & 6 & 100 & 2 & 5 & $113(11 \%)$ \\
\hline San Pedro & 0 & 29 & 29 & 23 & $81(8 \%)$ \\
\hline Penco & 31 & 27 & 0 & 19 & $77(7.6 \%)$ \\
\hline Tomé & 28 & & & & $28(2.7 \%)$ \\
\hline Hualpén & 51 & 2 & 27 & 10 & $90(8.9 \%)$ \\
\hline Coronel & 0 & 49 & 0 & 0 & $49(5 \%)$ \\
\hline Lota & 0 & 107 & 2 & 0 & $109(10.8 \%)$ \\
\hline Hualqui & 0 & 0 & 0 & 30 & $30(3 \%)$ \\
\hline Santa Juana & 0 & & & 20 & $20(2 \%)$ \\
\hline Totales & 172 & 490 & 122 & 226 & $1010(100 \%)$ \\
\hline
\end{tabular}


En la tabla 4 los estudiantes se distribuyen según sexo, se aprecia que los profesores nominaron un $10 \%$ más de hombres que de mujeres, situación que se repite, con leves diferencias, a través de los cuatro años.

\section{Tabla 4}

Frecuencias absolutas de nominados/as de la muestra según sexo del alumno/a nominado/a y año de preselección

\begin{tabular}{|l|r|r|r|c|c|}
\hline SEXO & 2007 & 2008 & 2009 & 2010 & Totales \\
\hline Mujeres & 79 & 222 & 55 & 101 & $457(45 \%)$ \\
\hline Hombres & 93 & 268 & 67 & 125 & $553(55 \%)$ \\
\hline Totales & 172 & 490 & 122 & 226 & $1010(100 \%)$ \\
\hline
\end{tabular}

En síntesis, la muestra tiene diversidad en cuanto a nivel educacional, aunque hay un predominio de sexto básico, como también respecto al sostenedor, a pesar de que está sub-representada la educación particular subvencionada y sobre-representada la municipal y la particular pagada. Las comunas están todas representadas y respecto al sexo hay un ligero predominio de los hombres.

Si bien el ser todos seleccionados por sus profesores como talentosos constituiría un sesgo, los puntajes Raven muestran amplia diversidad a este respecto; así, obtuvieron un promedio de 45.88 con una desviación estándar de 7.77, con un mínimo de 5 puntos y un máximo de 60. Al hacer un análisis de normalidad de la distribución de los puntajes Raven, se aprecia que siguen la curva normal. Por otra parte hay un $25 \%$ que está bajo 41 puntos, lo que corresponde al percentil 25. Para mostrar la variabilidad de los puntajes Raven se presentan los promedios, desviaciones estándar y puntajes mínimos y máximos por nivel educativo (Tabla 5). Se aprecia que en todos los niveles los promedios están en percentiles de normalidad y que, también en todos, hay puntajes que corresponden a inteligencias bajo lo normal. Por tanto podemos concluir que la muestra tiene diversidad respecto a la inteligencia de sus integrantes.

\section{Tabla 5}

Promedios, desviaciones estándar, mínimos y máximos de Raven por nivel educacional

\begin{tabular}{|l|c|c|c|c|}
\hline Promedios Raven (n) & Promedio & Desviación & Mínimo & Máximo \\
\hline Quinto (19) & 46.2 & 8.0 & 29 & 56 \\
\hline Sexto Básico (490) & 42.7 & 8.1 & 5 & 58 \\
\hline Séptimo (205) & 46.6 & 5.9 & 24 & 59 \\
\hline Octavo (107) & 48.0 & 5.4 & 35 & 60 \\
\hline Primero Medio (33) & 53.7 & 4.0 & 43 & 60 \\
\hline Segundo (57) & 52.4 & 4.6 & 43 & 60 \\
\hline Tercero (31) & 54.1 & 4.9 & 37 & 60 \\
\hline
\end{tabular}




\subsection{PROCEDIMIENTO}

La aplicación del instrumento se realizó en aulas de la Universidad de Concepción entre los meses de diciembre y abril de cada año, desde 2007 al 2010. El test es de auto respuesta y se aplicó en grupos de entre 20 y 30 sujetos. Para su aplicación se aprovechó la instancia de la asistencia a pruebas de selección de los alumnos postulantes al programa Talentos $\mathrm{U}$ de $\mathrm{C}$. Responder las preguntas de este cuestionario, de manera grupal, demora diez minutos.

\section{RESULTADOS}

Previo a la presentación de los resultados haremos un análisis sintético de la normalidad de la distribución de la variable, para luego presentar, primero, los aspectos psicométricos del cuestionario y posteriormente, la distribución de la adaptación socioemocional de los alumnos preseleccionados.

La distribución de la variable adaptación socioemocional es normal. La media fue de 3.99 , la mediana de 4.04 y la moda de 4.07 , con una desviación de 0.49 y un rango de 2.63. El perfil de la curva es el típico de la distribución normal, ligeramente inclinado a la derecha. La recta también corresponde a la normal, por tanto las pruebas estadísticas que se informarán son las adecuadas a una distribución normal.

En cuanto a la variación mostrada por los datos, ésta fue la máxima en todos los ítems. El promedio total fluctuó entre 2.37 y 5.00. Las desviaciones estándar de los ítems se movieron entre el 0.70 y 1.51 . Todo lo anterior muestra que el instrumento fue capaz de discriminar entre alumnos más adaptados y menos adaptados desde el punto de vista socioemocional.

\subsection{ANÁLISIS FACTORIAL}

Este análisis muestra claramente tres factores para el cuestionario, algo diferentes a los del instrumento original. Usando como límite aceptable para incluir el ítem una correlación de 0.40. De los 27 ítems originales hubo 4 que no se incluyeron en los tres factores: dos por correlación baja, que son el 5 (Siento que soy capaz de relacionarme con los adultos, de la subescala Habilidades sociales) y el 6 (Siento que mi rendimiento en la escuela no es bueno, originalmente de la subescala Habilidades cognitivas) y dos por aportar a dos factores, que son el ítem 7 (Soy una persona poco sociable, social) que aporta al factor I y al III, y el 23 (Cuando las cosas no salen como espero, dejo de intentarlo, de la subescala Habilidades emocionales) que aporta al factor I y al II.

De los tres factores, el primero explicaría el 3.73\% de la varianza, reuniría 10 ítems, sobre .40, quedando fuera el ítem el 23 y los ítems 6 y 26, este factor corresponde a habilidades emocionales. El factor 2 explica el $2.70 \%$ de la varianza, con ocho ítems, que hemos denominado de auto eficacia. Por último, el tercer factor explica un $2.40 \%$, con siete ítems, corresponde a la subescala de Habilidades sociales.

En la tabla siguiente presentamos los diez ítems del factor habilidades emocionales con sus respectivas correlaciones. 


\section{Tabla 6}

Correlaciones de los ítems que aportan al Factor 1 Habilidades emocionales

\begin{tabular}{|cl|c|}
\hline \multicolumn{1}{|c|}{ Ítem } & Correlación \\
\hline$* 2$ & Tengo dificultades para expresar lo que siento. & 0.62 \\
\hline$* 4$ & Suelo hacer cosas sin pensar en las consecuencias. & 0.51 \\
\hline$* 8$ & Prefiero estar solo en lugar de relacionarme con otras personas. & 0.46 \\
\hline$* 11$ & $\begin{array}{l}\text { Me resulta difícil encontrar la solución a los problemas que se me } \\
\text { presentan. }\end{array}$ & 0.62 \\
\hline$* 12$ & Me resulta difícil decir lo que pienso. & 0.67 \\
\hline$* 13$ & $\begin{array}{l}\text { Me he dado cuenta de que tengo dificultades para expresarme o } \\
\text { hablar en público. }\end{array}$ & 0.62 \\
\hline$* 16$ & Me cuesta acostumbrarme a los lugares nuevos para mi. & 0.59 \\
\hline$* 20$ & Me resulta difícil comprender a las personas y ponerme en su lugar. & 0.57 \\
\hline$* 26$ & Creo que las personas que me conocen no me aprecian demasiado. & 0.35 \\
\hline$* 27$ & Creo que no soy capaz de entender las cosas que siento. & 0.46 \\
\hline
\end{tabular}

* los ítems con asterisco son los invertidos, sus puntajes fueron revertidos, por tanto más altos promedios indican comportamientos adaptativos más adecuados.

En la tabla que sigue se presentan las correlaciones de los siete ítems que aportan al factor 2, que se ha denominado de autoeficacia.

\section{Tabla 7}

Correlaciones y clasificación por subescala de los ítems que aportan al factor 2 de percepción de Autoeficacia

\begin{tabular}{|cl|c|}
\hline \multicolumn{2}{|l|}{ Ítems } & Correlación \\
\hline 1 & Trabajo concentrado en una tarea hasta completarla. & 0.52 \\
\hline 15 & Me gusta intentar varias veces cuando algo no me resulta. & 0.64 \\
\hline 17 & $\begin{array}{l}\text { Algo que me gusta hacer es ayudar a otras personas cuando lo } \\
\text { necesitan. }\end{array}$ & 0.41 \\
\hline 19 & $\begin{array}{l}\text { Siento que las cosas que me propongo me resultan bien gracias a } \\
\text { mis capacidades. }\end{array}$ & 0.48 \\
\hline 22 & Cuando tengo un problema soy capaz de ver más de una solución. & 0.67 \\
\hline 24 & Cuando hago algo incorrecto me hago responsable de las consecuencias. & 0.42 \\
\hline 25 & Creo que soy una persona valiosa. & 0.48 \\
\hline
\end{tabular}


En la tabla siguiente se presentan las correlaciones de los ítems que aportan al factor 3 y que corresponden a habilidades sociales.

\section{Tabla 8}

\section{Correlaciones y clasificación por subescala de los ítems} que aportan al factor 3 de Habilidades sociales

\begin{tabular}{|c|c|}
\hline Ítems & Correlación \\
\hline 3 Suelo sentirme feliz & 0.51 \\
\hline 9 No tengo problemas para relacionarme con las personas de mi edad. & 0.50 \\
\hline \begin{tabular}{l}
$10 \begin{array}{l}\text { Mis compañeros me consideran como una persona a la que es impor- } \\
\text { tante invitar. }\end{array}$ \\
\hline $\begin{array}{l}14 \text { Me gusta participar en actividades como fiestas y reuniones de amigos } \\
\text { y compañeros. }\end{array}$
\end{tabular} & 0.51 \\
\hline 18 Me considero una persona alegre. & 0.58 \\
\hline 21 No siento vergüenza cuando tengo que hablar con gente de mi edad. & 0.42 \\
\hline
\end{tabular}

En síntesis se deben eliminar 2 ítems por baja correlación y 2 por aportar a dos factores, por tanto, la escala debería tener 23 ítems y tres subescalas que incluirán: 10 ítems en la subescala de Habilidades emocionales, 7 en la de Autoeficacia y 6 en la de Habilidades sociales.

\subsection{CONFIABILIDAD}

La escala de Adaptación socioemocional presentó con los 27 ítems un alfa de Cronbach de 0.85; con los 23 ítems que proponemos el alfa es de 0.84, cifras indicativas de consistencia interna alta.

Por otra parte, todos los ítems se correlacionaron directamente con el total de la escala de una manera altamente significativa (0.0001) como se puede apreciar en la tabla 9, con fuerzas de asociación que se mueven entre 0.31 y 0.56 , mayoritariamente sobre 0.40 . La dirección de las correlaciones de ítems también fue adecuada. Todo lo anterior demuestra confiabilidad de la escala. 
Tabla 9

Correlaciones $\mathrm{r}$ de Pearson ítem escala total de Adaptación socioemocional total $(\mathrm{n}=880)$

\begin{tabular}{|c|c|c|c|}
\hline Ítems Adaptación socioemocional & $\mathrm{r}$ de Pearson & $\mathrm{P}$ & Mínimos y máximos \\
\hline Item 1 & 0.34 & 0.0001 & 1.05 .0 \\
\hline Item 2 & 0.53 & 0.0001 & 1.05 .0 \\
\hline Item 3 & 0.37 & 0.0001 & 1.05 .0 \\
\hline Item 4 & 0.46 & 0.0001 & 1.05 .0 \\
\hline Item 5 & 0.46 & 0.0001 & 1.05 .0 \\
\hline Item 6 & 0.32 & 0.0001 & 1.05 .0 \\
\hline Item 7 & 0.53 & 0.0001 & 1.05 .0 \\
\hline Item 8 & 0.48 & 0.0001 & 1.05 .0 \\
\hline Item 9 & 0.37 & 0.0001 & 1.05 .0 \\
\hline Item 10 & 0.34 & 0.0001 & 1.05 .0 \\
\hline Item 11 & 0.49 & 0.0001 & 1.05 .0 \\
\hline Item 12 & 0.56 & 0.0001 & 1.05 .0 \\
\hline Item 13 & 0.56 & 0.0001 & 1.05 .0 \\
\hline Item 14 & 0.34 & 0.0001 & 1.05 .0 \\
\hline Item 15 & 0.39 & 0.0001 & 1.05 .0 \\
\hline Item 16 & 0.53 & 0.0001 & 1.05 .0 \\
\hline Item 17 & 0.31 & 0.0001 & 1.05 .0 \\
\hline Item 18 & 0.45 & 0.0001 & 1.05 .0 \\
\hline Item 19 & 0.42 & 0.0001 & 1.05 .0 \\
\hline Ítem 20 & 0.51 & 0.0001 & 1.05 .0 \\
\hline Ítem 21 & 0.36 & 0.0001 & 1.05 .0 \\
\hline Ítem 22 & 0.45 & 0.0001 & 1.05 .0 \\
\hline Ítem 23 & 0.54 & 0.0001 & 1.05 .0 \\
\hline Ítem 24 & 0.39 & 0.0001 & 1.05 .0 \\
\hline Ítem 25 & 0.38 & 0.0001 & 1.05 .0 \\
\hline Ítem 26 & 0.43 & 0.0001 & 1.05 .0 \\
\hline Ítem 27 & 0.46 & 0.0001 & 1.05 .0 \\
\hline
\end{tabular}

En cuanto a las subescalas, presentamos aquí los resultados con las dimensiones arrojadas por el análisis factorial; las subescalas se correlacionaron entre sí y con la escala total, de manera altamente significativas y con fuerza también alta, como se puede apreciar en la tabla que sigue, la número 10, lo que es un indicador más de la confiabilidad de la escala. 


\section{Tabla 10}

Intercorrelaciones subescalas escala total

\begin{tabular}{|l|c|c|c|c|}
\hline & $\begin{array}{c}\text { Adaptación } \\
\text { Total }\end{array}$ & Autoeficacia & $\begin{array}{c}\text { Habilidades } \\
\text { emocionales }\end{array}$ & $\begin{array}{c}\text { Habilidades } \\
\text { sociales }\end{array}$ \\
\hline Adaptación Total $(\mathrm{n}=882)$ & $\begin{array}{c}1.00 \\
<0.0001\end{array}$ & $\begin{array}{c}0.71 \\
<0.0001\end{array}$ & $\begin{array}{c}0.89 \\
<0.0001\end{array}$ & $\begin{array}{c}0.74 \\
<0.0001\end{array}$ \\
\hline Autoeficacia ( $\mathrm{n}=952)$ & & 1.00 & $\begin{array}{c}0.43 \\
<0.0001\end{array}$ & $\begin{array}{c}0.47 \\
<.0001\end{array}$ \\
\hline Habilidades emocionales (n=919) & & & 1.00 & $\begin{array}{c}0.55 \\
<.0001\end{array}$ \\
\hline Habilidades sociales $(\mathrm{n}=951)$ & & & & 1.00 \\
\hline
\end{tabular}

En relación a la consistencia interna de las subescalas, la que evalúa habilidades sociales presentó un Alfa de 0.76 y las correlaciones ítems subescala, que se presentan en la tabla que sigue, fueron todas positivas, fuertes y altamente significativas, por tanto se puede afirmar que esta subescala también es confiable.

\section{Tabla 11}

Correlaciones $\mathrm{r}$ de Pearson ítem total subescala de Habilidades sociales $(\mathrm{n}=951)$

\begin{tabular}{|c|c|c|}
\hline Ítems Adaptación social & $\mathrm{r}$ de Pearson & $\mathrm{p}$ \\
\hline Item 3 & 0.49 & 0.0001 \\
\hline Ítem 9 & 0.56 & 0.0001 \\
\hline Ítem 10 & 0.52 & 0.0001 \\
\hline Item 14 & 0.55 & 0.0001 \\
\hline Item 18 & 0.54 & 0.0001 \\
\hline Ítem 21 & 0.53 & 0.0001 \\
\hline
\end{tabular}

Respecto a la subescala que evalúa habilidades emocionales, ésta presentó un alfa de $0.84 \mathrm{y}$, como se puede observar en la tabla 12 , todos sus ítems se correlacionaron con el total de la subescala de manera altamente significativa y con una fuerza alta, mayoritariamente sobre 0.51 , por tanto también se puede afirmar que esta subescala es confiable para ser aplicada a niños, niñas y jóvenes de nuestro medio chileno. 
Tabla 12

Correlaciones $r$ de Pearson ítem total subescala de Habilidades emocionales $(n=919)$

\begin{tabular}{|c|c|c|}
\hline Ítems Adaptación emocional & $\mathrm{r}$ de Pearson & $\mathrm{p}$ \\
\hline Item 2 & 0.61 & 0.0001 \\
\hline Ítem 4 & 0.55 & 0.0001 \\
\hline Ítem 8 & 0.51 & 0.0001 \\
\hline Ítem 11 & 0.58 & 0.0001 \\
\hline Ítem 12 & 0.64 & 0.0001 \\
\hline Ítem 13 & 0.62 & 0.0001 \\
\hline Item 16 & 0.60 & 0.0001 \\
\hline Item 20 & 0.59 & 0.0001 \\
\hline Item 26 & 0.46 & 0.0001 \\
\hline Ítem 27 & 0.51 & 0.0001 \\
\hline
\end{tabular}

Por último, la subescala de auto eficacia presentó un alfa de Cronbach de 0.75, lo que está sobre el límite de confiabilidad aceptable; en cuanto a las correlaciones ítems subescala, que se observan en la tabla 13, todas son altamente significativas y con fuerzas altas, mayoritariamente sobre 0,51 , luego, esta subescala también es confiable.

\section{Tabla 13}

Correlaciones $r$ de Pearson ítem total subescala de Autoeficacia $(n=952)$

\begin{tabular}{|c|c|c|}
\hline Ítems Adaptación emocional & $\mathrm{r}$ de Pearson & $\mathrm{p}$ \\
\hline Item 1 & 0.53 & 0.0001 \\
\hline Ítem 15 & 0.53 & 0.0001 \\
\hline Ítem 17 & 0.45 & 0.0001 \\
\hline Ítem 19 & 0.58 & 0.0001 \\
\hline Ítem 22 & 0.62 & 0.0001 \\
\hline Ítem 24 & 0.51 & 0.0001 \\
\hline Ítem 25 & 0.56 & 0.0001 \\
\hline
\end{tabular}

Todo lo anterior permite afirmar que el Cuestionario de Adaptación Socioemocional para niños, niñas y jóvenes es un instrumento confiable para ser aplicado en nuestra realidad chilena. 


\subsection{VALIDEZ}

En primer lugar, la escala fue elaborada basándose en la experiencia de psicólogos infanto-juveniles, lo que sería una garantía de validez constructiva.

En segundo lugar, trescientos once profesores de los estudiantes de la muestra respondieron un cuestionario acerca de la adaptación socioemocional de éstos. El cuestionario para profesores también fue elaborado por los psicólogos de Talentos $\mathrm{U}$ de $\mathrm{C}^{2}$. Consta de diecinueve ítems, de ellos ocho son invertidos y tiene el mismo formato que el de los alumnos. Los datos obtenidos con este instrumento permiten evaluar la validez convergente del cuestionario para niños/as. Hubo correlaciones positivas significativas entre la adaptación de los alumnos informada por los profesores y la autopercepción de adaptación total $(\mathrm{r}=0.13, \mathrm{p}<0.02)$ y altamente significativas con la subescala Habilidades emocionales $(\mathrm{r}=0.14, \mathrm{p}<0.009)$. Aunque estas correlaciones son indicativas de validez son menores a las esperadas. Estas bajas correlaciones tal vez se podrían explicar porque los profesores evaluaron como más alta la Adaptación socioemocional de sus alumnos/ as, con un promedio de 4.5 frente al 4.0 de los alumnos.

En cuanto a los hallazgos que son similares a los de la literatura, hubo diferencias significativas de Adaptación socioemocional según el sexo de los alumnos; las mujeres tuvieron un promedio de 4.1 significativamente superior al 3.9 de los hombres $(t=4.91$, $\mathrm{p}<0.0001)$. Diferencias que se repiten a favor de las mujeres en dos subescalas, Habilidades sociales y emocionales $(\mathrm{r}=3.07, \mathrm{p}<.002 \mathrm{y} \mathrm{r}=4.71, \mathrm{p}<0.0001$, respectivamente). Esto coincide con resultados presentados por otros autores como Siverio y García (2007) y Garaigordobil (2005), que informan mayor inadaptación en niños que en niñas. Siverio y García (2007) reportan mayores conductas antisociales en los adolescentes varones, lo que es indicativo también de diferencias de género en el mismo sentido encontrado por nosotros. Lo anterior mostraría la validez de la escala y de dos de sus subescalas. En cuanto a la tercera subescala, los hombres tienen un promedio de percepción de su autoeficacia significativamente mayor que el de las mujeres $(t=4.74, p<0.0001)$ lo que coincidiría con lo informado por Olaz (2003). En relación a la edad, hubo correlación positiva, aunque baja, de la edad con la Adaptación socioemocional $(r=0.09$, $\mathrm{p}<0.007$ y más fuerte con subescala Habilidades emocionales $(\mathrm{r}=0.16, \mathrm{p}<0.0001)$, esto es concordante con lo esperable del proceso de madurez y lo planteado por Gardner (2005) en relación con su concepto de la matriz del talento. Además, Siverio y García (2007) informan mayor inadaptación social en niños que en adolescentes, lo que tendría el mismo sentido que a mayor edad mayor adaptación, puesto que nuestra muestra tiene niños y niñas entre 9 y 19 años.

\subsection{ADAPTACIÓN SOCIOEMOCIONAL DE LOS ALUMNOS DE LA MUESTRA}

El promedio de Adaptación socioemocional fue de 4.00 con una desviación de 0.49 y varió entre 2.37 y 5 , que es el puntaje máximo posible; el promedio en Habilidades sociales fue de 4.2 con una desviación de 0.55 y fluctuó entre 1.9 y 5; la Adaptación emocional tuvo un promedio de 3.58 , con una desviación de 0.73 y varió entre 1.18 y 5. Por último, el promedio de Autoeficacia fue de 4.36, con una desviación de 0.46 y

2 No existen publicaciones al respecto 
un mínimo de 2.25 y un máximo de cinco (5). En la tabla que sigue se presentan los promedios y desviaciones por ítems. Se aprecia que en general la adaptación socioemocional es alta, considerando que el máximo posible es 5. De los 27 ítems, 13 quedan sobre cuatro puntos y sólo 4 bajo dos puntos.

\section{Tabla 14}

Adaptación socioemocional de los alumnos/as de la muestra por ítems

\begin{tabular}{|c|c|c|}
\hline Ítem & Promedio & Desviación \\
\hline 1- Trabajo concentrado en una tarea hasta completarla. & 4.5 & 0.7 \\
\hline *2- Tengo dificultades para expresar lo que siento. & 2.9 & 1.4 \\
\hline 3- Suelo sentirme feliz. & 4.4 & 0.8 \\
\hline *4- Suelo hacer cosa sin pensar en las consecuencias. & 2.5 & 1.3 \\
\hline 5- Siento que soy capaz de relacionarme con adultos. & 4.1 & 1.1 \\
\hline *6- Siento que mi rendimiento escolar no es bueno. & 1.7 & 1.1 \\
\hline *7- Soy una persona poco sociable. & 1.9 & 1.1 \\
\hline *8- Prefiero estar solo en lugar de con otras personas. & 1.8 & 1.2 \\
\hline 9- No tengo problemas para relacionarme con las personas de mi edad. & 4.3 & 1.1 \\
\hline 10- Mis compañeros me consideran como una persona a la que es importante invitar. & 3.7 & 1.1 \\
\hline *11- Me resulta difícil encontrar la solución a los problemas que se me presentan. & 2.4 & 1.2 \\
\hline *12- Me resulta difícil decir lo que pienso. & 2.4 & 1.3 \\
\hline *13- Me he dado cuenta de que tengo dificultades para expresarme o hablar en público. & 2.6 & 1.5 \\
\hline 14- Me gusta participar en actividades como fiestas y reuniones de amigos y compañeros. & 4.3 & 1.1 \\
\hline 15- Me gusta intentar varias veces cuando algo no me resulta. & 4.5 & 0.8 \\
\hline *16- Me cuesta acostumbrarme a los lugares nuevos para mi. & 2.9 & 1.4 \\
\hline 17- Algo que me gusta hacer es ayudar a otras personas cuando lo necesitan. & 4.4 & 0.8 \\
\hline 18- Me considero una persona alegre. & 4.5 & 0.8 \\
\hline 19- Siento que las cosas que me propongo me resultan bien gracias a mis capacidades. & 4.4 & 0.8 \\
\hline *20- Me resulta difícil comprender a las personas y ponerme en su lugar. & 2.6 & 1.3 \\
\hline 21- No siento vergüenza cuando tengo que hablar con gente de mi edad. & 4.2 & 1.2 \\
\hline 22- Cuando tengo un problema soy capaz de ver más de una solución. & 4.2 & 0.9 \\
\hline *23- Cuando las cosas no salen como espero, dejo de intentarlo. & 1.9 & 1.1 \\
\hline 24- Cuando hago algo incorrecto me hago responsable de las consecuencias. & 4.4 & 0.9 \\
\hline 25- Creo que soy una persona valiosa. & 4.3 & 0.9 \\
\hline *26- Creo que las personas que me conocen no me aprecian demasiado. & 2.1 & 1.2 \\
\hline *27- Creo que no soy capaz de entender las cosas que siento. & 2.5 & 1.5 \\
\hline
\end{tabular}

* Los ítems con asterisco son los invertidos, sus puntajes fueron revertidos, por tanto más altos promedios indican comportamientos adaptativos más adecuados. 
Los puntajes más altos, con 4,5 puntos de promedio, corresponden a: el ítem 1 (Trabajo concentrado), el 15 (Me gusta intentar varias veces cuando algo no me resulta) y el 18 (Me considero una persona alegre). Le siguen, en orden decreciente con 4.4, el ítem 3 (Suelo sentirme feliz), el 17 (Algo que me gusta hacer es ayudar a otras personas cuando lo necesitan), el 19 (Siento que las cosas que me propongo me resultan bien gracias a mis capacidades) y el 24 (Cuando hago algo incorrecto me hago responsable de las consecuencias).

Los puntajes más bajos corresponden a los ítems 6 (Siento que mi rendimiento en la escuela es bueno), 7 (Soy una persona poco sociable), 8 (Prefiero estar solo en lugar de relacionarme con otras personas) y 23 (Cuando las cosas no salen como espero, dejo de intentarlo). Llama la atención que los bajos promedios se concentren en los ítems invertidos, lo que podría atribuirse a dificultades de algunos alumnos/as para comprenderlos; sin embargo, como ya se vio, estos ítems mostraron altas correlaciones con la escala total y la subescala correspondiente, indicativas de su alta confiabilidad.

\section{CONCLUSIONES}

La primera conclusión es que este instrumento presenta tres factores que no corresponden exactamente a las tres subescalas originales, por lo tanto deben reclasificarse algunos ítems y eliminarse otros. Eliminados los cuatro que presentaron problemas de confiabilidad de acuerdo a los parámetros establecidos en esta investigación, la escala queda de 23 ítems distribuidos en tres subescalas. Así, de la subescala de Habilidades emocionales quedan 6 de sus 11 ítems originales, se elimina uno (el 5) y pasan a esta subescala 3 ítems de la subescala de Habilidades sociales $(8,12$ y 13 ) y uno de la de Habilidades cognitivas (el 11), por tanto la nueva subescala Habilidades reúne 10 ítems. La subescala de Habilidades cognitivas es reemplazada por la de Autoeficacia, que queda con 7 ítems, tres de habilidades cognitivas (1, 15 y 22), tres de habilidades incluidas en la escala original como emocionales $(19,24$ y 25$)$ y uno de la subescala de Habilidades sociales (el 17). Por último, la nueva escala incluye en su subescala de Habilidades sociales seis ítems: 4 de la subescala original $(9,10,14$ y 21) y dos clasificados anteriormente como emocionales (3 y el 18).

Una segunda conclusión es que este cuestionario es un instrumento confiable, así lo demuestran el alfa de Cronbach total y por subescalas, las correlaciones ítem escala e ítem subescala correspondiente y las intercorrelaciones escala total subescalas.

La inspección de su validez refleja, por la vía de correlacionar el cuestionario respondido por los alumnos/as con el respondido por sus profesores, validez convergente. Las diferencias entre hombres y mujeres, que muestran a las mujeres con promedios de adaptación mayores en Habilidades sociales y emocionales y a los hombres con promedio mayor de percepción de Autoeficacia, concuerdan con lo informado en la literatura (Olaz, 2003), y por tanto, también son indicativas de validez.

En general, la adaptación socioemocional de los alumnos y alumnas preseleccionados al programa fue alta. Particularmente éste fue el caso en Autoeficacia, lo que es esperable, dado que se trata de niños elegidos por sus profesores como con talento académico destacado. Le sigue muy cerca el promedio de Habilidades sociales y bastante más bajo el de Habilidades emocionales, lo que también es concordante con lo esperado para esta etapa de la vida. 
Con el análisis realizado, estimamos que el instrumento presentado puede constituir un aporte a la investigación del desarrollo socioemocional en jóvenes, niños y niñas de nuestro medio.

\section{REFERENCIAS BIBLIOGRÁFICAS}

Bralic, S. y Romagnoli, C. (2000). Niños y jóvenes con talento: una educación de calidad para todos. Dolmen: Santiago de Chile.

Gagné, F. (2000). Un modelo diferenciador de dotación y talento. Notas personales traducidas por Sonia Bralic (con autorización del autor). Université du Québec à Montreal: Canada.

Gardner, H. (2005). Inteligencias múltiples. La teoría en la práctica. Paidos: Barcelona, $1^{\text {a }}$ edición.

De la Cruz, M.V. y Cordero, A. (1997). Inventario de Adaptación de Conducta (IAC). Madrid: TEA ediciones.

Garaigordobil, M. (2005). Conducta antisocial durante la adolescencia: Correlatos Socioemocionales, predictores y diferencias de género. Psicología Conductual, 13(2), 197-215.

Mathiesen M.E., Castro, G., Merino, J.M., Mora, O. y Navarro G. (2010-2011). Incubando capital humano regional: evaluación longitudinal de un programa universitario para estudiantes de establecimientos municipales de alto potencial académico. Proyecto Fondecyt $\mathrm{N}^{\circ} 1100260$.

Olaz, F. (2003). Autoeficacia, diferencias de género y comportamiento vocacional. Revista electrónica de motivación y emoción, 6(13). Recuperado en marzo de 2011 de http://reme.uji.es/ articulos/aolazf5731104103/texto.html

Siverio E., Miguel A. y García, M. (2007). Autopercepción de adaptación y tristeza en adolescentes: Influencia del género. Anales de Psicología 23(1), 41-48. Publicación de la Universidad de Murcia: España. 
